ARTIGO ORIGINAL ORIGINAL ARTICLE

\title{
O impacto económico da prevenção de úlceras de pressão num hospital universitário
}

\author{
The economic impact of pressure ulcers \\ prevention in a university hospital \\ Bruno Venâncio ${ }^{1}$, Eduardo Alves², Catarina Ruano 3 , Daniela Matos 4 , \\ Sara Valente ${ }^{5}$, Nuno Abreu ${ }^{6}$, Renato Mota ${ }^{7}$ \\ DOI: 10.21115/JBES.v11.n1.p64-72
}

\section{Palavras-chave:}

úlceras de pressão, internamento hospitalar, ganhos em saúde, prevenção, idosos

\section{Keywords:}

pressure ulcers, length of stay, elderly, health gains, prevention

\section{RESUMO}

Objetivo: As úlceras de pressão constituem uma condição evitável que se traduz numa diminuição da qualidade de vida do doente. Simultaneamente representam um acréscimo nos custos para os serviços de saúde, associados ao aumento do tempo de internamento. A investigação é fundamental para justificar a prevenção enquanto medida de sustentabilidade e melhoria dos cuidados de saúde. O estudo visa estimar o efeito individual das diferentes categorias das úlceras de pressão no incremento do tempo de internamento hospitalar e os ganhos em saúde que derivam da intervenções autónomas e independentes dos enfermeiros do domínio da prevenção das UP. Métodos: A determinação do impacto incremental das diferentes categorias das UP no tempo de internamento é obtida por uso de um modelo econométrico concebido para esse fim. Os ganhos em saúde são determinados com o uso da equação preconizada pela ordem profissional dos enfermeiros (OE). Resultados: $\mathrm{O}$ impacto incremental das diferentes categorias de UP no internamento hospitalar é por ordem crescente de severidade clínica da UP de 1,05 dias; 1,64 dias; 3,53 dias e 10,29 dias. Estima-se que tenha sido prevenido o incremento de 2977,61 dias de internamento hospitalar, em resultado da prevenção das úlceras de pressão. Conclusão: É possível incrementar os ganhos em saúde e contribuir na redução do impacto das UP no internamento hospitalar ao clinicamente indispensável através da maximização na efetividade diagnóstica.

\begin{abstract}
Objective: Pressure ulcers are an avoidable condition that results in a decrease in the health related quality of life of the client. At the same time, they represent an increase in costs for health services associated with an increase in length of hospital stay. Research in this area is fundamental to justify prevention as a measure of sustainability and improvement of health care. The study aims to estimate the individual effect of the different categories of pressure ulcers on the increase of hospitalization time and the health gains derived from autonomous and independent interventions of nurses in the field of PU prevention. Methods: The determination of the incremental impact of the different UP categories in the length of stay is obtained by using an econometric model designed for this purpose. Health gains are determined using the equation recommended by the professional order of nurses (OE). $\mathbf{R e}$ sults: The incremental impact of the different categories of PU on hospital admission is in ascending order of clinical severity of PU of 1.05 days; 1.64 days; 3.53 days and 10.29 days. It is estimated that an increase of 2977.61 days of hospitalization was prevented, as a result of the prevention of pressure ulcers. Conclusion: It is possible to increase the health gains and contribute in reducing the impact of PUs in hospital admission to the clinically indispensable by maximizing the diagnostic effectiveness.
\end{abstract}

\footnotetext{
Recebido em: 07/03/2019. Aprovado para publicação em: 17/04/2019.

1. Urgência Geral, CHUP, Porto, Portugal.

2. Conselho de Administração, CHUP, Porto, Portugal.

3. Cirurgia Geral, CHUP, Porto, Portugal.

4. Clínica Cirúrgica, CHUP, Porto, Portugal.

5. CMIN-CHUP, Porto, Portugal.

6. Sistemas de Informação e Gestão, CHUP, Porto, Portugal.

7. Cirurgia Vascular, CHUP, Porto, Portugal.

Fontes de financiamento: Não existiram fontes de financiamento para o estudo realizado.

Local onde o estudo foi desenvolvido: $O$ estudo foi desenvolvido no Centro Hospitalar Universitário do Porto, E.P.E.

Declaração de conflitos de interesse dos autores: Os autores não apresentam qualquer tipo de conflitos de interesse. Autor correspondente: Bruno Manuel de Sousa Venâncio. Centro Hospitalar Universitário do Porto (CHP), Urgência Geral. Largo Prof. Abel Salazar, Porto, Portugal. CEP: 4099-001. Telefone: + 351 918566483. E-mail: bruno.venancio.urg@chporto.min-saude.pt / enf.brunovenancio@gmail.com
} 


\section{Introdução}

O tempo de internamento consiste numa medida da efetividade dos cuidados de saúde, refletindo, em parte, a qualidade dos serviços prestados pelas instituições e sistemas de saúde (Theisen et al., 2012; Vetrano et al., 2014). As instituições, com um aumento do tempo de internamento, além do tempo médio expectado, para uma dada condição clínica apresentam um consequente aumento evitável dos custos e também uma perda significativa da capacidade hospitalar. Esta última é medida pela taxa de ocupação das camas no período excedente, ou seja, as mesmas durante esse período seriam utilizadas no tratamento de outros doentes, constituindo isto o custo de oportunidade.

O incremento do período de internamento está associado, no caso dos doentes idosos, a uma aceleração do declínio funcional e à crescente probabilidade de desenvolvimento de complicações hospitalares (i.e.: infeções nosocomiais, úlceras de pressão, entre outros) (Graves et al., 2005; Theisen et al., 2012; Vetrano et al., 2014).

Embora o diagnóstico médico principal constitua o maior preditor do tempo de internamento hospitalar, há uma diversidade de fatores que influenciam este último, sobretudo na população envelhecida (Graves et al., 2005; Vetrano et al., 2014).

Uma parte significativa dos fatores identificados é do domínio clínico, mas não na sua totalidade, existindo outros de natureza social e económica (não clínicos) que apresentam um papel relevante.

No que diz respeito aos fatores clínicos, os que estabelecem uma associação estatisticamente significativa com o aumento do tempo de internamento são o maior número de doenças crónicas, um maior declínio cognitivo e funcional, a capacidade de marcha preservada com velocidade de deslocação inferior a $0.80 \mathrm{~m} / \mathrm{s}$, a dependência em atividades de vida diária e a excessiva polimedicação (ingestão de $10 \mathrm{ou}$ mais medicamentos), em que este último constituiu um dos preditores com maior impacto (Graves et al., 2005; Vetrano et al., 2014). Do mesmo modo, uma maior pontuação no charlson comorbidity index (CCl) está associada a um maior incremento do tempo de internamento (Vetrano et al., 2014).

Uma parte significativa dos fatores anteriormente citados traduz-se em necessidades de apoio no domicílio. Em contextos sociais e económicos onde o suporte familiar e social do doente é frágil ou inexistente para suprimir as mesmas, ocorre um incremento do tempo de internamento em consequência do protelamento da alta clínica (Wright et al., 2003). A referenciação para os cuidados continuados de doentes que necessitam de reabilitação funcional após um episódio agudo de doença constitui também, no Hospital em análise, um fator associado ao aumento do tempo de internamento (Ferreira, 2012).

Alguns estudos concluem que determinados fatores, como a permanência em unidades de cuidados intensivos (UCI), o uso de ventilação invasiva e infeções, encontram-se associados a um incremento do tempo de internamento, tal como a presença da sonda nasogástrica e a incontinência fecal. Essas variáveis não são fatores explicativos do aumento do tempo de internamento, mas são definidas como variáveis proxys que representam determinados estados de saúde. O primeiro conjunto de fatores descrito define doentes com um pior estado de saúde e uma admissão hospitalar potencialmente complicada, o segundo conjunto representa um grupo de comorbilidades dos doentes que se encontravam omissas no modelo de regressão multivariado (Graves et al., 2005; Theisen et al., 2012).

As úlceras de pressão são lesões da pele ou tecido subjacente localizadas, normalmente, sobre as proeminências ósseas e contribuem para um aumento significativo da estadia hospitalar causando um impacto variável entre os 2,6 e os 16 dias (Allman et al., 1999; Ash, 2002; Gomes et al., 2014; Graves et al., 2005; Gunningberg \& Stotts, 2008; Schuurman et al., 2009; Theisen et al., 2012; Williams et al., 2000). Não obstante, a incidência hospitalar de UP, em doentes de cirurgia major do domínio cardiotorácico, demonstrou que o impacto incremental no tempo de internamento não foi estatisticamente significativo, mas contribuiu para um aumento considerável no tempo de permanência em UCl. Estes últimos ficaram 7,4 dias em UCl, um valor comparativamente superior aos 4,1 dias apresentado em doentes sem úlcera de pressão. Estas foram o único fator explicativo com significância estatística para o sucedido (Schuurman et al., 2009). Por sua vez, alguns autores referem que apenas 5,5\% da incidência hospitalar de UP é responsável pelo aumento do tempo de internamento, permanecendo os doentes no hospital apenas para o exclusivo tratamento das mesmas (Lardenoye et al., 2009).

A prevalência de UP na admissão hospitalar alcança os $22,9 \%$, enquanto que a incidência varia entre $1,3 \%$ e os $53,4 \%$ em resultado das características clínicas e do contexto de cuidados (Gunningberg \& Stotts, 2008; Lardenoye et al., 2009; Schuurman et al., 2009). No contexto português, um estudo realizado no Hospital de Aveiro, em 2012, revelou que 3,4\% dos doentes apresentaram UP, em que 63,5\% foram desenvolvidas em contexto hospitalar (Sardo et al., 2016). As UCl e as enfermarias de Ortopedia e de Medicina Interna apresentam os valores de incidência, normalmente, mais acentuados (Graves et al., 2005). Contudo, o impacto da prevalência e da incidência é distinguível no tempo de internamento. Verifique que o impacto do primeiro estimou-se em 13,8 dias, embora significativo é claramente inferior aos 24,5 dias consequente do segundo. A odd ratio dos doentes que desenvolvem UP no hospital incrementarem a estadia hospitalar é de 1,62 em contraste com 1,24 da prevalência de UP na admissão (Theisen et al., 2012).

O aumento dos custos que deriva do prolongamento da estadia hospitalar resulta, em particular, do tempo despendido pelos enfermeiros com o tratamento das UP, da atividade diag- 
nóstica do risco e consequente aplicação de medidas de prevenção (i.e.: posicionamento, entre outros), e da utilização de material de consumo clínico e farmacêutico (MCCF) (Bennett et al., 2004; Theisen et al., 2012). A probabilidade de transição para estados de saúde de severidade clínica superior ou com complicações na cicatrização que derivam da colonização, celulites e osteomielites contribui para a dilatação dos custos. $\bigcirc$ tratamento da UP de categoria IV não complicada e uma outra com osteomielite apresenta um custo que se estende entre 7.750 e 24.214 libras, respetivamente. O custo médio do tratamento da UP de categoria I à IV, englobando as probabilidades de complicações, oscila entre 1.064 e 10.571 libras. Estimou-se que o gasto anual com o tratamento de UP correspondeu a 4,1\% da despesa total em saúde no Reino Unido, em que 90\% da mesma corresponde ao tempo dos profissionais de enfermagem (Bennett et al., 2004).

Na perspetiva do doente, as UP incrementam a mortalidade e a morbilidade (i.e.: dor, desconforto, diminuição da funcionalidade e da independência) e também o desenvolvimento de complicações hospitalares (Lardenoye et al., 2009; Theisen et al., 2012). O impacto das mesmas na qualidade de vida relacionada com a saúde (QVRS) imputa uma desutilidade entre os 0,028 e 0,029 (Essex et al., 2009; Soares et al., 2013).

Os idosos são a população que mais UP desenvolvem no hospital, totalizando 70\% dos novos casos de UP, e decorrem com maior frequência em doentes com internamento de natureza cirúrgica (Graves et al., 2005; Schuurman et al., 2009; Theisen et al., 2012). Relativamente a este último estima-se que a condição clínica de internamento é o maior preditor de risco para desenvolver UP. Observe que, em casos de fratura da extremidade proximal do fémur (FEPF) e amputação dos membros inferiores, a incidência alcançou os $10 \%$ e os 8,8\%, respetivamente. No mesmo estudo, a incidência hospitalar numa enfermaria de cirurgia geral foi de apenas 1,3\% (Lardenoye et al., 2009; Vetrano et al., 2014).

Outros fatores que se encontram associados ao desenvolvimento de UP no hospital são a malnutrição, a imobilização prolongada, a incontinência fecal e urinária e também a lacuna nos cuidados de prevenção (Graves et al., 2005; Lardenoye et al., 2009). Um estudo demonstrou que a prestação de cuidados em desacordo com as recomendações do European Pressure Ulcer Advisory Panel (EPUAP) estava associada a acentuados valores de incidência no pós-operatório de cirurgia major em UCl (Schuurman et al., 2009).

Observa-se que 49,5\% da incidência hospitalar das UP decorreu nas primeiras 24 horas, e apenas 55,2\% dos doentes que as desenvolveram tinham sido identificados com Risco de UP (Gunningberg \& Stotts, 2008; Schuurman et al., 2009). Sublinhando-se ainda que apenas 31,3\% dos casos de UP resultaram diretamente das comorbilidades do doente, apesar destes últimos as apresentarem frequentemente em maior número (Lardenoye et al., 2009).
A incidência hospitalar de UP é comumente utilizada como um indicador institucional, reflexo da qualidade dos cuidados de enfermagem, e a sua utilização promove maior eficiência e segurança para o doente. A sua aplicação constitui-se também de grande interesse pela sua fiabilidade que é resultante das UP estarem bem definidas e serem de fácil identificação e registo. A sua utilização, enquanto, medida de comparabilidade da qualidade dos cuidados institucionais de enfermagem deve ser ajustado pelo mix-case (comorbilidades dos doentes) e o diagnóstico clínico de internamento (Gunningberg \& Stotts, 2008; Lardenoye et al., 2009).

Uma melhoria no desempenho deste indicador traduz-se em ganhos numa perspetiva agregada, como, por exemplo, na redução do internamento hospitalar ao clinicamente indispensável. Este último associado a uma otimização do consumo de recursos e consequentemente redução dos custos no Serviço Nacional de Saúde (SNS) (Theisen et al., 2012; Vetrano et al., 2014).

\section{Métodos}

\section{Objetivos}

I) Estimar a taxa de efetividade diagnóstica do risco de úlcera de pressão em doentes idosos ( $\geq 65$ anos) num Hospital Universitário;

II) Estimar a taxa de efetividade da prevenção de complicações hospitalares, em particular, das úlceras de pressão em doentes idosos ( $\geq 65$ anos) num Hospital Universitário;

III) Estimar a incidência das úlceras de pressão por categoria em doentes idosos ( $\geq 65$ anos), num Hospital Universitário.

IV) Estimar se as úlceras de pressão são um preditor independente e estatisticamente significativo do incremento do tempo de internamento, mesmo que ajustado pela severidade clínica, complexidade e outros fatores relevantes do episódio de assistência;

V) Quantificar o impacto da incidência de úlceras de pressão no incremento do tempo de internamento;

VI) Estimar os ganhos em saúde em resultado da prevenção das úlceras de pressão.

\section{Hipótese de investigação}

I) As úlceras de pressão são um fator explicativo independente e estatisticamente significativo do incremento do tempo de internamento.

II) As intervenções de enfermagem no domínio da prevenção das úlceras de pressão estão associadas à diminuição do tempo de internamento hospitalar.

\section{Amostra}

É um estudo retrospetivo, com os dados dos sistemas de informação e gestão provenientes do registo clínico dos profissionais de saúde. Os critérios de inclusão incluem episódios de assistência em que os doentes apresentam: a) admissão hospitalar 
posterior a 1 de janeiro de 2016 e alta clínica anterior a 30 de setembro do mesmo ano; b) idade igual ou superior a 65 anos e; c) tempo de internamento igual ou superior a 1 dia. Os critérios de exclusão retiram episódios de assistência em que os doentes apresentam: a) prevalência de úlcera de pressão na admissão hospitalar com categoria igual ou superior a Il; b) úlcera de pressão com categoria indefinida, uma vez que, se encontram incluídos, de acordo com a instituição em estudo, casos com úlcera de pressão em que a informação do registo clínico foi insuficiente para classificar adequadamente a categoria; c) episódios cuja informação é insuficiente quanto à unidade em que se encontravam internados e; d) informação insuficiente sobre a natureza de admissão (urgente/programada), situação clínica (médica/ cirúrgica), classificação no Grupo de Diagnósticos Homogéneos $(\mathrm{GDH})$ e respetivo nível de severidade.

\section{Variáveis}

No Hospital Universitário em estudo, a mensuração do risco, a prevenção e o tratamento das úlceras de pressão decorrem, de acordo, com as recomendações de um procedimento geral e interno, intitulado "Gestão integrada da prevenção e risco de úlcera de pressão" codificado pelo gabinete da governação clínica com a sigla PG.GGC.GER.011/0 (Centro Hospitalar Universitário do Porto, 2016). A mensuração do risco de úlcera de pressão é comumente efetuada no contexto da atividade diagnóstica do enfermeiro, atendendo frequentemente ao uso de um instrumento auxiliar, a escala de Braden adaptada à realidade portuguesa (Braden \& Bergstrom, 1994; Direcção-Geral da Saúde, 2011). A classificação das úlceras de pressão é efetuada, de acordo, com os critérios de diagnósticos recomendados pela European Pressure Ulcer Advisory Panel (EPUAP) (Gomes et al., 2014).

A efetividade diagnóstica do risco de úlcera de pressão e da prevenção das úlceras de pressão é calculada através de indicadores internos, tendo este sido adaptado das recomendações emitidas pela Ordem dos Enfermeiros (OE) (Ordem dos Enfermeiros de Portugal, 2007). A efetividade diagnóstica consiste no número de doentes internados que evoluem para um estado de saúde em que apresentam úlcera de pressão, encontrando-se identificado previamente no processo de cuidados eletrónico (PCE), o diagnóstico de enfermagem do risco de úlcera de pressão. A efetividade na prevenção de complicações de úlceras de pressão consiste no número de doentes internados que, identificados com o diagnóstico de enfermagem de risco de úlcera de pressão, não evoluíram para um estado de saúde que apresentasse a mesma.

Os doentes com risco de úlcera de pressão representam os doentes com fatores de risco inerentes ao seu estado de saúde, que incrementam a probabilidade de desenvolver, durante o episódio de assistência, úlceras de pressão. Recebendo no período imediato, estratégia personalizada na prevenção das mesmas (Braden \& Bergstrom, 1994). A probabilidade cres- cente é resultado, do diagnóstico de internamento, das características individuais do doente, das comorbilidades e das respetivas sequelas (Lardenoye et al., 2009).

O registo clínico de úlceras de pressão que decorre num período inferior às 24 horas após admissão hospitalar é considerado pela instituição em estudo prevalência na admissão. Isto é, as úlceras de pressão não foram desenvolvidas no contexto hospitalar, em oposição, todos os registos clínicos que decorrem após esse período são considerados incidência. Em cada episódio de assistência foi contabilizado por doente apenas a úlcera de pressão cuja categoria refletia a maior severidade clínica e não foram distinguidos a presença de múltiplas úlceras em diferentes localizações anatómicas (Centro Hospitalar Universitário do Porto, 2016). As úlceras de pressão são uma variável qualitativa ordinal em que admite quatro opções: I) UP-I; II) UP-II; III) UP-III e IV) UP-IV.

O tempo de internamento (TI) é mensurado em dias e consiste na subtração entre a data de admissão e alta clínica ou a morte do doente. Tendo em consideração a variabilidade do case-mix da população em estudo, calculou-se o tempo de internamento ajustado (TIA). Esta última é a subtração entre o valor atual e a média do tempo de internamento expectado para o GDH e nível de severidade em que o doente foi correspondentemente classificado. Os doentes que apresentam um tempo de internamento ajustado (TIA) com valor negativo significam que permanecem no hospital menos tempo do que a média nacional.

O peso do GDH é uma variável contínua que reflete a complexidade e a severidade da condição clínica, afeta ao doente, ou seja, quanto maior o valor do mesmo, maior a severidade e consequente quantidade de recursos consumida (Busse, 2011; Mihailovic et al., 2016). Por seu turno, o peso médio é um conceito semelhante ao índice de case-mix, mas não incorpora a conversão da produção em doentes equivalente. Assim, o mesmo reflete a complexidade da casuística de internamento da amostra em estudo (Ministério da Saúde, 2015; Santana, 2005).

A referenciação de um doente pela Equipa de Gestão de Altas (EGA) representa, na instituição em estudo, o doente que necessita de cuidados de saúde após o período da alta clínica, mas permanece na mesma, a aguardar a vaga para a Rede Nacional de Cuidados Continuados Integrados (RNCCl) (Ferreira, 2012). É uma variável binária, que assume o valor de 1, caso o doente tenha sido referenciado e 0, caso contrário.

A variável "destino da alta" é uma variável qualitativa, que representa o destino da alta dos doentes, admitindo-se quatro possibilidade: I) Cuidados Continuados Integrados (CCl); II) internamento num outro hospital (Transferência), III) Falecimento e, IV) outro.

\section{Análise estatística}

A análise bivariada foi efetuada para avaliar as diferenças estatisticamente significativas no tempo de internamento ajustado 
(TIA) entre doentes com e sem úlcera de pressão. E, também, estimar a associação entre outros fatores explicativos, referidos na literatura, e a influência no tempo de internamento. Os testes estatísticos utilizados foram os não paramétricos, nomeadamente, o teste de Wilcoxon e o Kruskal-Wallis. Os intervalos de confiança foram calculados para um nível de confiança a 95\%, e para as amostras que não apresentam um n, suficientemente grande, recorre-se por prudência, à distribuição com n-1 graus de liberdade, obtendo-se intervalos com amplitude ligeiramente maior.

Na determinação da significância e independência das úlceras de pressão, enquanto preditor do incremento do tempo de internamento foi desenvolvido um modelo de regressão linear múltiplo (MRLM). Adicionou-se uma constante à variável TIA, de modo a converter a totalidade dos valores em números positivos e, consequentemente, executou-se a transformação logarítmica natural da mesma, obtendo-se a Log (TIAC). O Modelo de regressão foi testado como um todo, testando-se a significância global e a significância individual, das variáveis explicativas, através da apresentação da estatística $F$ e $t$, respetivamente, para um nível de significância de 5\% ( $p<0,05)$. Os pressupostos do modelo foram testados, nomeadamente, o da normalidade, da homocedasticidade e da autocorrelação dos resíduos, mas também quanto à multicolinearidade das variáveis explicativas. Aplicaram-se, respetivamente, os seguintes testes: o teste Jarque Bera (JB), Teste de White (TW), estatística da Durbin-Watson (DW), para os dois primeiros, o nível de significância foi de 5\% ( $p<0,05)$. Quanto à multicolinearidade, utilizou-se a estatística FIV (Variance Inflation Factor), esperando-se uma regressão aceitável para valores menores que 5.

Todos os testes foram executados com o Eviews 9.0, tendo-se aplicado, para o caso da presença de autocorrelação e heterocedasticidade, a correção de Newey-West, que corrige os erros padrão dos coeficientes tornando-os consistentes.

\section{Resultados}

Entre 1 de janeiro de 2016 e o 31 de setembro do mesmo ano, apenas 8.274 episódios de assistência corresponderam aos critérios de elegibilidade para integrar o estudo. A média de idades e o respetivo desvio padrão foi de 76,94 \pm 8,02 anos; dos quais $51,09 \%$ são do sexo feminino. A média do tempo de internamento da amostra em estudo é de 8,76 \pm 11,19 dias, variando o mesmo entre 1 e os 218 dias, com uma mediana de 5 dias. Por sua vez, o Tempo de Internamento Ajustado (TIA) variou entre os -67,89 e os 173,31 dias, apresentando uma média de $-1,14 \pm 10,07$ dias, refletindo a mesma um valor inferior à média nacional. Apenas 28,79\% (2382) dos doentes em estudo apresentavam um tempo de internamento superior ao expectado. Em média, os doentes com úlcera de pressão apresentam um tempo de internamento não ajustado superior em 14,58 dias e 2,4 vezes maior, face aos que não as desenvolvem.
Os fatores estatisticamente associados com o incremento do TIA, na análise bivariada ( $p<0,05)$, incluem o risco e a incidência de úlcera de pressão e a referenciação pela EGA. A natureza da admissão e o peso médio crescente apresentam uma associação estatisticamente significativa ( $p<0,05)$, mas de relação inversa. Denote que os doentes que apresentam severidade clínica superior detêm um tempo de internamento inferior à média nacional. Não obstante, a média do tempo de internamento não ajustado pelos graus de severidade é progressivamente mais acentuada com os níveis crescente da mesma. Os grupos etários, o sexo, as categorias das úlceras de pressão e, ainda, a situação clínica não apresentam associação significativa $(p<0,05)$ com o incremento do TIA (Tabela 1).

Os doentes que apresentam úlcera de pressão refletem um peso médio superior $(2,27 \pm 3,56)$ face aos que não as desenvolvem $(1,30 \pm 1,57)$. Observe que o peso médio capta a complexidade e a severidade clínica dos doentes em cada episódio de assistência. Esta resulta da exaustividade do registo clínico dos profissionais de saúde, quanto ao diagnóstico principal, secundário, complicações e co-morbilidades do episódio. Assim, assume-se que um maior valor justifica um episódio com maior gravidade e consequentemente um maior tempo de internamento, de complicações, de mortalidade e de readmissões.

As categorias das úlceras de pressão desenvolvidas em contexto hospitalar são um preditor independente e estatisticamente significativo do incremento do tempo de internamento. O grupo omisso no modelo são doentes sem úlcera de pressão, sem referenciação da EGA e com alta clínica, o domicílio (Tabela 2).

Um doente que desenvolve úlcera de pressão de categoria I e, de acordo, com o Modelo - I, apresenta um tempo de internamento, em média, 9,07\% superior a um outro doente do grupo base, coeteris paribus. $\mathrm{O}$ impacto é crescente com a severidade clínica da úlcera de pressão, em que se estima que na categoria II, III e IV um impacto percentual de $13,61 \%, 22,28 \%$ e 55,99\%, respetivamente. O impacto incremental, em dias, das diferentes categorias das úlceras de pressão, encontra-se apresentado na tabela 3. A perpetuação do sinal negativo na variável, peso relativo, significa que o Hospital Universitário em estudo apresenta um desempenho, comparativamente melhor, face à média nacional, no tratamento de doentes com crescente complexidade clínica.

O modelo II representa a subamostra de doente que apresentava o diagnóstico de risco de úlcera de pressão e o grupo omisso apresenta as mesmas características que o do modelo I. Contudo, o impacto incremental das diferentes categorias de UP no internamento hospitalar é por ordem crescente de severidade clínica da UP, 1,05 dias; 1,64 dias; 3,53 dias e 10,29 dias.

No Hospital Universitário em estudo, a proporção dos doentes com risco de úlcera de pressão foi de 19,82\%, contudo, a incidência de úlcera de pressão foi de 2,99\% e não de- 
correu exclusivamente em doentes com o risco previamente identificado. Assim, resultou numa média hospitalar de efetividade diagnóstica de 55,87\%, enquanto que a efetividade na prevenção foi de 91,59\% (Tabela 4). Tendo em consideração os valores observados anteriormente, estimou-se que a inefetividade diagnóstica resultou numa perda 45,48 dias. Esta última foi calculada ajustada pela categoria de maior severidade clínica da UP no doente.

Os ganhos em saúde resultantes da taxa de efetividade diagnóstica e de prevenção, atendendo aos diferentes valores de incidência em consonância com as categorias de UP e assumindo que os doentes com risco desenvolveriam obrigatoriamente UP em internamento hospitalar, no caso da ausência de medidas de prevenção, foram obtidos em função da equação abaixo indicada.

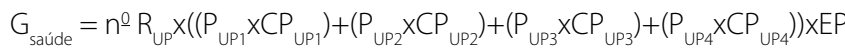

Equação 1. Ganhos em saúde na prevenção de úlceras de pressão

Legenda:

$n^{\circ} R_{u p}-$ Número total de doentes identificados com risco de úlcera de pressão

EP - Taxa de efetividade das medidas de prevenção das úlceras de pressão

$\mathrm{P}_{\text {uP }}$-Probabilidade de desenvolver UP

$\mathrm{CP}_{\text {UP }}$-Número incremental de dias de internamento em função da categoria de UP

Por outras palavras, assume-se que sem intervenção de prevenção todos os doentes desenvolveriam UP. Sendo que a incidência estimada por categoria é igual ao número de doen-

Tabela 1. Tempo de Internamento Ajustado (TIA) por características do episódio de assistência

\begin{tabular}{|c|c|c|c|c|c|c|}
\hline & $n$ & Média & Desvio Padrão & Mediana & IC & Valor $p$ \\
\hline $\begin{array}{l}\text { Úlcera Pressão } \\
\text { (UP) } \\
\text { Sim } \\
\text { Não }\end{array}$ & $\begin{array}{c}247 \\
8027\end{array}$ & $\begin{array}{r}8,09 \\
-1,42\end{array}$ & $\begin{array}{c}21,69 \\
9,35\end{array}$ & $\begin{array}{c}2,5 \\
-2,16\end{array}$ & $\begin{array}{l}{[5,39 ; 10,79]} \\
{[-1,62 ;-1,22]}\end{array}$ & 0,0000 \\
\hline $\begin{array}{l}\text { Referenciação } \\
\text { EGA } \\
\text { Sim } \\
\text { Não }\end{array}$ & $\begin{array}{c}158 \\
8116\end{array}$ & $\begin{array}{l}17,36 \\
-1,50\end{array}$ & $\begin{array}{c}25,04 \\
9,19\end{array}$ & $\begin{array}{l}12,85 \\
-2,16\end{array}$ & $\begin{array}{c}{[13,46 ; 21,26]} \\
{[-1,70 ;-1,30]}\end{array}$ & 0,0000 \\
\hline $\begin{array}{l}\text { Categoria UP } \\
1 \\
2 \\
3 \\
4\end{array}$ & $\begin{array}{l}117 \\
91 \\
31 \\
8\end{array}$ & $\begin{array}{c}5,50 \\
8,69 \\
14,20 \\
15,38\end{array}$ & $\begin{array}{l}20,71 \\
20,93 \\
23,57 \\
32,74\end{array}$ & $\begin{array}{c}0,92 \\
3,25 \\
10,27 \\
3,055\end{array}$ & $\begin{array}{c}*[1,73 ; 9,27] \\
*[4,37 ; 13,01] \\
*[5,77 ; 22,63] \\
*[-8,87 ; 39,63]\end{array}$ & 0,2026 \\
\hline $\begin{array}{l}\text { Natureza } \\
\text { admissão } \\
\text { Urgente } \\
\text { Programada }\end{array}$ & $\begin{array}{l}5291 \\
2983\end{array}$ & $\begin{array}{l}-0,57 \\
-2,15\end{array}$ & $\begin{array}{l}11,57 \\
6,49\end{array}$ & $\begin{array}{l}-2,21 \\
-2,13\end{array}$ & $\begin{array}{l}{[-0,88 ;-0,26]} \\
{[-2,38 ;-1,92]}\end{array}$ & 0,0296 \\
\hline
\end{tabular}


tes em cada categoria a dividir pelo número total de doentes com UP, isto na subamostra de doentes com risco de úlcera de pressão.

Na substituição das incógnitas da equação n.o 1 pelos valores do Modelo II, estima-se que tenha sido prevenido o incremento de 2977,61 dias de internamento hospitalar, em resultado da prevenção das úlceras de pressão.

\section{Discussão de resultados}

A mensuração dos ganhos em saúde foi efetuada em consonância com o modelo equacional n. ${ }^{\circ}$ 1, tendo sido construído com base na informação documental apresentada pela ordem dos enfermeiros (Ordem dos Enfermeiros de Portugal, 2007).

Tabela 2. Modelo de regressão linear múltiplo

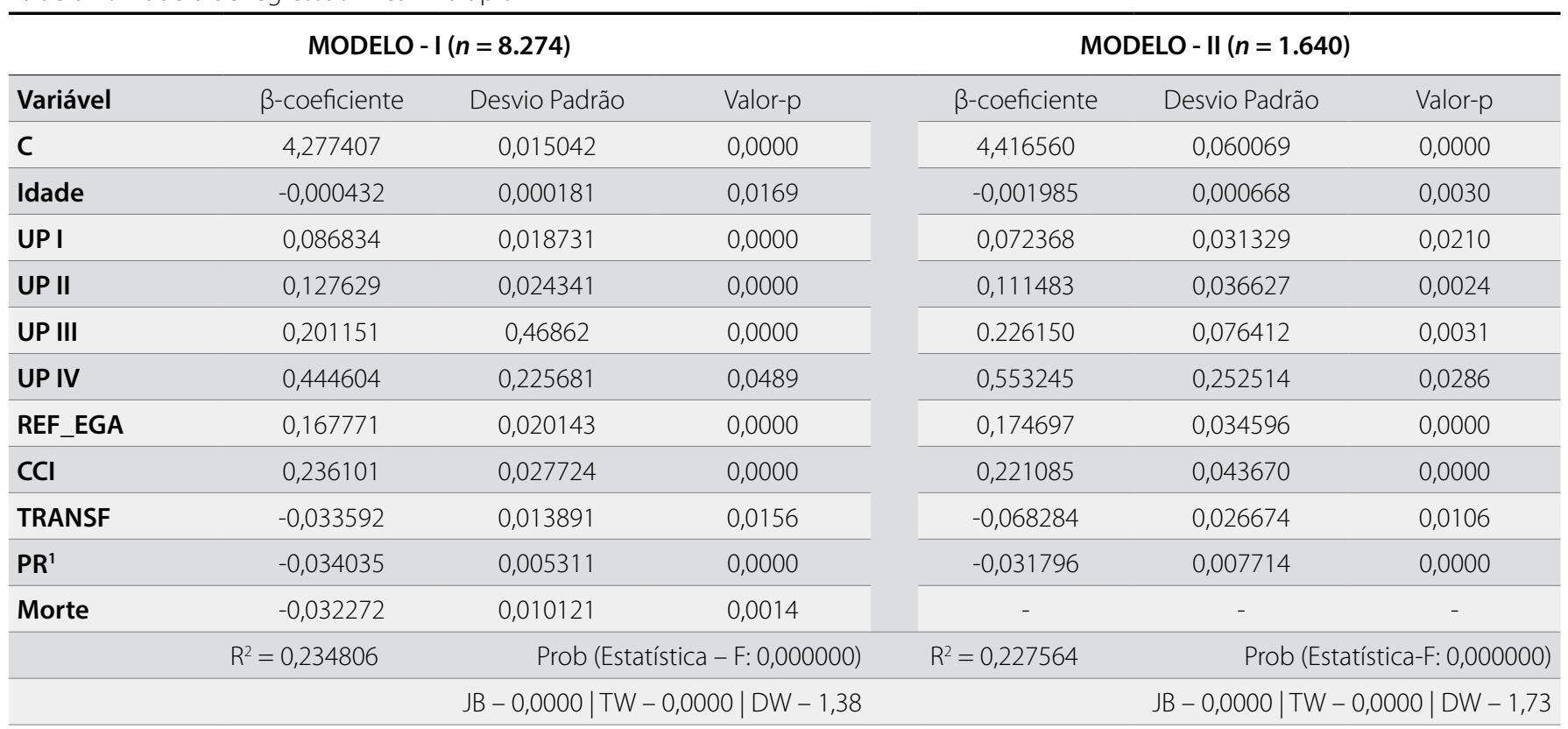

* Log (TIAC) - Logaritmo natural da variável, tempo de internamento ajustado com adição de constante.

Legenda: 1 - Peso Relativo.

Tabela 3. Impacto das categorias de UP no tempo de internamento hospitalar

\begin{tabular}{ccccc}
\hline Categoria & Impacto incremental (dias) & N & Custo perdido (dias) & 33,93 \\
\hline I & 0,29 & 117 & 39,13 \\
II & 0,43 & 91 & 21,7 \\
III & 0,70 & 31 & 14,16 \\
IV & 1,77 & 8 & 108,92 \\
\hline TOTAL & - & 247 & \\
\hline
\end{tabular}

Tabela 4. Resultados dos indicadores de desempenho interno

\begin{tabular}{lccccc}
\hline DEPARTAMENTOS* & $\boldsymbol{n}$ & Risco UP & Úlcera Pressão (UP) & $\begin{array}{c}\text { Efetividade } \\
\text { diagnóstica }\end{array}$ & $\begin{array}{c}\text { Efetividade } \\
\text { prevenção }\end{array}$ \\
\hline Medicina & 3.100 & $21,65 \%$ & $4,45 \%$ & $48,55 \%$ & $90,01 \%$ \\
\hline Cirurgia & 2.134 & $14,15 \%$ & $1,45 \%$ & $58,06 \%$ & $94,04 \%$ \\
\hline I \& E & 178 & $45,51 \%$ & $6,18 \%$ & $63,64 \%$ & $91,36 \%$ \\
\hline Ortofisiatria & 872 & $36,81 \%$ & $5,39 \%$ & $63,83 \%$ & $90,65 \%$ \\
\hline Neurociências & 1.088 & $15,62 \%$ & $1,75 \%$ & $78,95 \%$ & $91,76 \%$ \\
\hline Hospital & 8.274 & $19,82 \%$ & $2,99 \%$ & $55,87 \%$ & $91,59 \%$ \\
\hline
\end{tabular}

* Apenas se encontram representados os departamentos em que se verificam incidências de úlceras de pressão.

${ }^{1}$ Anestesiologia, Cuidados Intensivos e Emergência. 
No entanto, a equação é passível de melhorias, uma vez que a mesma induz uma sobrestimação dos ganhos mensurados. A fórmula tem por base o pressuposto de que todos os doentes identificados com risco desenvolveriam UP na ausência de intervenção no domínio da prevenção, mas não é verdade, dado que a probabilidade é variável de doente para doente e, por certo, existem doentes que não desenvolveriam UP, apesar de terem sido identificados com o risco de UP. Assim, recomendar-se-ia que no cálculo dos ganhos em saúde o valor da efetividade das medidas de prevenção fosse estimado pela diferença da incidência de UP em doente com risco sem intervenção e a incidência de UP em doentes com risco e intervenção. No caso em estudo, não foi possível reproduzir esse cálculo, pela ausência dos dados sobre o primeiro cenário. No entanto, os valores obtidos no MRLM II traduzem o impacto das UP no tempo de internamento após a implementação de medidas de prevenção e tratamento. Em que o impacto descrito é significativamente inferior ao impacto que se observaria na ausência da intervenção. Deduzindo-se, assim, que o incremento do internamento hospitalar seria significativamente superior, tal como o observado em diferentes estudos (Allman et al., 1999; Ash, 2002; Graves et al., 2005; Gunningberg \& Stotts, 2008; Schuurman et al., 2009; Theisen et al., 2012; Williams et al., 2000). A probabilidade dos doentes com risco de desenvolverem diferentes categorias de UP de severidade cínica superior é significativamente inferior ao que se esperaria sem medidas de prevenção e tratamento. Logo, os ganhos em saúde mensurados encontram-se também eles significativamente subestimados pelos dois fatores anteriormente citados.

Quanto aos valores de incidência de UP em doentes sem risco previamente identificado refletem a taxa efetividade diagnóstica, esta última poderá ser potencialmente melhorada no futuro e tais melhorias traduzir-se-iam em ganhos em saúde adicionais. Constate que, apesar do referido, os valores identificados neste estudo são ligeiramente melhores do que apresentados em estudos prévios, $58,87 \%$ versus os $44,8 \%$ (Gunningberg \& Stotts, 2008).

As UP são um preditor estatisticamente significativo e independente do incremento do internamento hospitalar, admitindo-se uma conclusão semelhante a estudos previamente analisados. Contudo, as categorias de UP exerceram um impacto estatisticamente significativo, independente e incrementalmente crescente com a severidade clínica da UP. Contrariamente, em diversos estudos, não conseguiram evidenciar esta relação, apesar dos diversos autores terem expectado teoricamente este resultado (Schuurman et al., 2009; Theisen et al., 2012).

$O R^{2}$ de ambos os modelos apresentados, apesar de ser baixo, encontra-se próximo e é por vezes superior ao de alguns estudos que tiveram o mesmo objeto de estudo e uma metodologia relativamente semelhante (Graves et al., 2005; Theisen et al., 2012).

\section{Conclusão}

O incremento da severidade clínica das úlceras de pressão exerce um impacto crescente estatisticamente significativo e independente no incremento do tempo de internamento além do clinicamente necessário para a condição clínica de internamento.

A identificação dos doentes com risco de úlcera de pressão através da atividade diagnóstica efetuada pelos enfermeiros e a implementação de medidas prevenção personalizadas, no Hospital Universitário em estudo, proporcionou ganhos em saúde, medido pelo incremento de número de dias de internamento hospitalar evitados, de 2.977,61 dias.

É possível incrementar os ganhos em saúde e contribuir na redução do impacto das UP no internamento hospitalar ao clinicamente indispensável através da maximização na efetividade diagnóstica.

\section{Limitações do estudo}

os resultados de investigação são afetados pela exaustividade do registo clínico efetuado pelos profissionais de saúde.

O período da amostra em estudo foi condicionado atendendo a mudanças organizacionais que resultaram na integração do ICD-10CM, pelo que não foi possível estender o período da amostra até aos dias de hoje.

A integração de um software para a governação clínica e a consequente interoperabilidade e tradução das bases de dados não permitiram o estudo referente ao período prévio a 2016.

\section{Referências bibliográficas}

Allman RM, Goode PS, Burst N, Bartolucci AA, Thomas DR. Pressure ulcers, hospital complications, and disease severity: impact on hospital costs and length of stay. Adv Wound Care. 1999;12(1):22-30.

Ash D. An exploration of the occurrence of pressure ulcers in a British spinal injuries unit. J Clin Nurs. 2002;11(4):470-8.

Bennett $G$, Dealey C, Posnett J. The cost of pressure ulcers in the UK. Age Ageing. 2004;33(3):230-5.

Braden BJ, Bergstrom N. Predictive validity of the Braden Scale for pressure sore risk in a nursing home population. Res Nurs Health. 1994;17(6):459-70.

Busse R, Geissler A, Quentin W, Wiley M. Diagnosis-Related Groups in Europe Moving towards transparency, efficiency and quality in hospitals. Open University Press; 2011.

Centro Hospitalar Universitário do Porto. Gestão integrada de prevenção e risco de úlcera de pressão; 2016.

Direcção-Geral da Saúde. Escala de Braden: Versão Adulto e Pediátrica. Orientação da Direção-Geral da Saúde, 1-10; 2011. Available at: http:// www.dgs.pt/. Accessed on: 05/14/2019.

Essex HN, Clark M, Sims J, Warriner A, Cullum N. Health-related quality of life in hospital inpatients with pressure ulceration: Assessment using generic health-related quality of life measures. Wound Repair Regen. 2009;17(6):797-805.

Ferreira LGO. O efeito da referenciação de utentes para a Rede Nacional de Cuidados Continuados Integrados sobre a demora média dos internamentos no Centro Hospitalar do Porto, 112; 2012. 
Gomes FSL, Bastos MAR, Matozinhos FP, Temponi HR, Velasquez-Melendez G, Pereira AGS, et al. Prevenção e Tratamento de Úlceras por Pressão: Guia de Consulta Rápida. Revista da Escola de Enfermagem. 2014;43. Available at: https://doi.org/10.1016/j.brachy.2016.04.297. Accessed on: 05/14/2019.

Graves N, Birrell F, Whitby M. Effect of pressure ulcers on length of hospital stay. Infect Control Hosp Epidemiol. 2005;26(3):293-7.

Gunningberg L, Stotts NA. Tracking quality over time: what do pressure ulcer data show? Int J Qual Health Care. 2008;20(4):246-53.

Lardenoye JW, Thiéfaine JA, Breslau PJ. Assessment of incidence, cause, and consequences of pressure ulcers to evaluate quality of provided care. Dermatol Surg. 2009:35(11):1797-803.

Mihailovic N, Kocic S, Jakovljevic M. Review of Diagnosis-Related Group-Based Financing of Hospital Care. Health Serv Res Manag Epidemiol. 2016 May 12;3:2333392816647892.

Ministério da Saúde. (2015). Portaria n 234/2015. Diário da República, 153, 5516-5654

Ordem dos Enfermeiros de Portugal. (2007). Sistemas de Informação de Enfermagem (SIE): Resumo Mínimo de Dados e Core de Indicadores de Enfermagem para o Repositório Central de Dados da Saúde. Documentos oficiais-2007. [acesso em: 13 abr 2013]. Available at: http://www.esenfcvpoa. eu/wp-content/uploads/2012/03/RMDE.pdf. Accessed on: 05/14/2019.

Santana R. O financiamento hospitalar e a definição de preços. Revista Portuguesa de Saúde Pública. 2005;93-118.
Sardo PM, Simões CS, Alvarelhão JJ, Simões JF, Machado PA, Amado FM, et al. Analyses of pressure ulcer incidence in inpatient setting in a Portuguese hospital. J Tissue Viability. 2016;25(4):209-15.

Schuurman JP, Schoonhoven L, Keller BP, van Ramshorst B. Do pressure ulcers influence length of hospital stay in surgical cardiothoracic patients? A prospective evaluation. J Clin Nurs. 2009;18(17):2456-63.

Soares MO, Dumville JC, Ashby RL, Iglesias CP, Bojke L, Adderley U, et al. Methods to assess cost-effectiveness and value of further research when data are sparse: negative-pressure wound therapy for severe pressure ulcers. Med Decis Making. 2013;33(3):415-36.

Theisen S, Drabik A, Stock S. Pressure ulcers in older hospitalised patients and its impact on length of stay: a retrospective observational study. J Clin Nurs. 2012;21(3-4):380-7.

Vetrano DL, Landi F, De Buyser SL, Carfì A, Zuccalà G, Petrovic M, et al. Predictors of length of hospital stay among older adults admitted to acute care wards: A multicentre observational study. Eur J Intern Med. 2014;25(1):56-62.

Williams DF, Stotts NA, Nelson K. Patients with existing pressure ulcers admitted to acute care. J Wound Ostomy Continence Nurs. 2000;27(4):216-26.

Wright SP, Verouhis D, Gamble G, Swedberg K, Sharpe N, Doughty RN. Factors influencing the length of hospital stay of patients with heart failure. Eur J Heart Fail. 2003;5(2):201-9. 